\title{
Belching, CTCAE
}

National Cancer Institute

\section{Source}

National Cancer Institute. Belching, CT CAE. NCI Thesaurus. Code C143198.

To expel gas noisily from the mouth. 